\title{
Author index to volume 34
}

Key to abbreviations:

(BR) Book review; (E) Editorial; (L) Letter; (O) Obituary notice; (R) Review

\author{
Aalberg V 669 \\ Abe E 615 \\ Abe J 220 \\ Açikgöz B 361 \\ Addy M 498 (L) \\ Adkins RH 188, 707 \\ Aggarwal A 346 \\ Akaboshi K 531 \\ Al-Ali M 34 \\ Alcaraz MA 164 \\ Allen SJ 193 \\ Ament PA 107 \\ Amodie-Storey C 602 \\ Andrews BJ 8 \\ Annen S 427, 699 \\ Asazuma T 620 \\ Ashley E A 264 \\ Awanda A 630 \\ Aydoğan N 297 \\ Azuchi M 427
}

Baba H 100, 427, 644, 699

Babhulkar SS 716

Bae HG 176

Baker HWG 696

Bamford ES 752

Banyard PJ 449 (R)

Barber DB 173

Barraclough BM 752

Batra KM 346

Baysefer A 297

Bazzini G 684

Beard JP 173

Bennie A 560

Bergström E 560

Bernard P-L 288

Betz R 127

Betz RR 16

Biering-Sørensen F 666

Bishop GR 82

Boake C 193

Boden B 127

Bodley R 65, 180 (L)

Boileau G 95

Borg J 204

Bourne H 696

Bowsher D 707

Brackin B 82

Bravo P 164

Briggs DE 338

Brooks CA 470

Brown DJ 277

Bulmer CE 639

Burnham R 264

Bussel B 91
Byrne DW 255

Cairbre McCann B 433

Cameron KJ 277

Campbell IG 659

Çamurdanoğlu M 361

Canakçi Z 297

Cao A 305

Capodaglio P 684

Cardenas DD 239

Castelló T 592

Castelló-Verdú T 315

Chattopadhyay 633

Chino N 531

Chow YW 736

Cila A 301

Clapper A 214

Clifton GL 193

Cohen ME 543

Cohen MJ 107

Collier GR 277

Comarr AE (deceased) 501 (O)

Condie DN 611

Crane LD 602

Crossman MW 573

Cruse JM 82

Cumming C 351

Cumming D 351

Cumming DC 264

Cumming J 93

Curt A 326

Dallmeijer AJ 729

Darouiche RO 742

Davie MWJ 736

de Mello MT 294

Dehoux E 630

Del Valle-Ortiz O 315

Delahaye $\mathrm{H} 95$

Di Biasi C 720

Dickson RA 566 (BR), 567 (BR)

Dietz V 326

Dimitrijevic MM 193

Dincer F 748

Ditunno JF Jr 543

Doh JW 176

Dollfus P 187 (Presidential Message)

Domen K 531

Donovan WH 193, 587

Ebata K 615

Edwards J 659
Edwards JV 193

Eisma WH 679

El Masry W 736

Ellis ER 54

Eltorai I 501

Erbengi A 358

Erdoğan E 297

Fiegel VD 394

Fife R 127

Foley SJ 657

Foroglou G 268

Fortuna A 720

Frances Jones HW 565

Frankel H 65

Fraser MH 137, 578, 745

Froelich JW 338

Frost RA 180 (L)

Fujimaki E 394

Fujimura Y 387, 620

Fukuzawa K 234

Furusawa N 427, 644

García L 592

García-Fernández L 315

Gardner B 65

Gardner E 127

Garfin SR 113

Girona L 592

Glasby M 181 (BR)

Golman SJ (deceased) 59 (O)

Gómez MR 592

Gönül E 297

Granat MH 8, 611

Grassino A 204, 594

Grilli C 684

Groothoff JW 679

Grundy DJ 30, 93, 752

Güler-Uysal F 301

Gurjar SG 333

Haas BM 560

Haddad L 34

Haddaway MJ 736

Haghighi SS 39, 214

Hamada Y 234

Handa Y 411, 699

Harata S 486

Harber V 264

Harper GP 449 (R)

Harris EC 752

Harvey LA 54

Hashimoto T 411

Hatter E 308

Hattori T 481
Hawran S 666

Hayami S 411

Heller BW 8

Herbison GJ 543

Hermens HJ 507

Hirabayashi K 620

Hiraizumi Y 394

Hirao Y 493

Hirata N 493

Hirayama A 493

Hockley AD 179

Hopman MTE 729

Horvat JC 320

Hoshi K 725

Hoshino Y 725

Hourani F 308

Howard CV 137

Hull R 587

Hultling C 152

Hurley ME 113

Ichikawa S 486

Ikata T 234, 381 (E), 416, 536

Illis LS 566 (BR), 568 (BR)

Imura S 100, 427, 644, 699

Inci S 358

Inman C 736

Inoue A 220, 536

Inskip HM 752

Isaac Z 543

Ishigooka M 411

Ishihara K 422

Ishikawa $\mathrm{H} 422$

Ito $\mathrm{J} 486$

Iwatsubo E 416

Jamous A 560

Janzen J 498 (L), 499 (L)

Johnson RL 470

Joshi A 742

Jung V 351

Kagaya H 24, 615

Kalita J 272

Kamitani K 427

Kannisto M 669

Karamehmetoglu SS 498 (L)

Karavelis A 268

Katoh D 234

Katoh S 234, 416, 536

Kawabata H 422

Kawahara N 100, 394

Kawauchi Y 403

Kayali H 297

Kinoshita H 1 
Klefbeck B 167

Kliesch WF 82

Klingensmith WC III 338

Knighton D 394

Knox AW 602

Krausher R 351

Krishnan KR 121 (L), 137, 243, 499 (L), 578, 651, 691, 745, 754 (L)

Ku A 116, 193

Kunkel C 46

Kurokawa T 725

Labarta C 164

Lachmann E 116

Lammertse DP 338

Lampton JA 82

Lancashire P 498 (L)

Laskin JJ 264

Lauro FAA 294

Le Gallais D 288

Lee KS 176

Lee SM 284

Lefkoe TP 239

Levi R 152

Lewis RE 82

Lewko JP 158

Lim T 696

Lindström L 204, 594

Lissens MA 673

LoMonaco M 488

Loubser PG 755 (L)

Lunardi P 720

McKinley WO 626

MacLeod GM 525

Macleod L 525

McSweeney T (deceased) 565 (O)

Maezawa Y 100, 427, 644, 699

Maharaj JC 549

Maruo S 382

Mastronardi L 720

Matsuzaki H 422

Mattsson E 167

Matumoto M 382

Maurice-Williams RS 568 (BR)

Mehta S 633

Meisheri YV 633

Mena Mur A 592

Mendoza J 164

Menter RR 59

Mesgarzadeh M 127

Meyer C 466

Micallef J-P 288

Minns RA 460

Misra UK 272

Mitra KR 333

Mitra SR 333

Miyoshi K 725

Moissonnier P 320

Momose H 493

Moody FG 193
Morita M 220

Mulcahey MJ 16

Mulder GA 679

Nagata K 220

Nagata M 531

Nagler W 116

Nakada T 411

Nakamura K 725

Nakamura M 387

Nash MS 602

Natsume O 493

Nene AV 507

Nishi Y 387

Nyulasi IB 277

Oba M 615

Ohashi T 220

Okajima E 493

Okumura Y 644

Olenik LM 264

Olona-Cabases M 315

Önol B 358

Onomura T 422

Orkun S 301, 748

Oro JJ 39

Osman Farah J 720

Ota T 531

Otom A 308

Oxenham D 567 (BR)

Özgen T 361

Özgirgin N 301

Padua L 488

Padua R 488

Pajareya K 608

Palaoğlu S 358

Palkar V 630

Pande KC 716

Pande SK 716

Parsons KF 137, 243, 578

Pau AC 305

Paul JP 611

Perell K 46

Perez-Espejo MA 39

Perez-Espejo M-A 214

Perkash I 227, 501

Perrigot M 95

Petsanas A 268

Pickard WG 30

Pollintine P 736

Porru D 305

Potts JR III 193

Poulain M 288

Powell ES 659

Prévinaire JG 95

Pringle RG 181 (BR), 498 (L)

Puzzilli F 720

Quine S 639

Ramírez-Garcerán L 315

Rémy Néris O 91

Reviron T 320

Roberts JF 338
Roby-Brami A 91

Rodriguez F 214

Ross ERS 659

Rossi CD 587

Roussell PM 602

Rowley DI 611

Saita K 725

Sakakibara R 481

Sakou T 403

Salzberg CA 255

Sammallahti P 669

Sangwan SS 346

Sannohe A 486

Sato K 24, 615

Sato M 24, 615

Sato T 486

Satomi K 620

Scarpa RM 305

Schandler SL 107

Schönherr MC 679

Schumacker P 95

Scremin A 46

Scremin O 46

Scuderi GJ 113

Seiger $\AA 152$

Seki M 531

Sett P 65

Shah PJR 657

Sharp CA 736

Sharpe PC 449 (R)

Sheriff MKM 657

Sherwood AM 193

Shimada Y 24, 615

Shingu H 416

Shiomi T 493

Sie I 188

Sie IH 707

Silva AC 294

Silver JR 60 (L), 122 (L), 499 (L)

Sinderby C 204, 594

Siwach RC 346

Sloley D 264

Smith BT 16

Smith WI 338

Snoecx M 500 (L)

Soler JM 95

Soni BM 137, 243, 578, 651, 691,745

Sonoda S 531

Sözay S 748

Spollen L 39

Steadward R 351

Steadward RD 264

Stien R 365

Sullivan L (deceased) 204, 365 (O), 594

Sung JH 394

Sungur C 361

Sutherland JD 338

Suzuki N 387, 620

Suzuki Y 411

Sykes L 659

Szollar SM 284
Tanaka Y 493

Tator CH 60 (L)

Tian W 486

Ticó-Falguera N 315

Tomita K 100, 427

Tonali PA 488

Toyama Y 387

Transfeldt EE 394

Trasimeni G 720

Triolo R 127

Tromans AM 93

Tufik S 294

Tunkel R 116

Uchida K 427, 644, 699

Ueyama K 486

Usai E 305

Vaccaro AR 113

Vaidyanathan S 121 (L), 137, 243, 499 (L), 578, 651, $691,745,754(\mathrm{~L})$

van As HHJ 729

van der Woude LHV 729

Van Do S 755 (L)

van Velzen D 137, 243, 578

Vanderstraeten GG 673

Vanhée JL 95

Vanvelcenaher J 95

Varray A 288

Verdú A 164

Vinet A 288

Vulpe M 107

Wade WH 173

Wakabayashi K 422

Wang D 65

Waters RL 188, 707

Watkins W 696

Watt JWH 691

Weinberg J 167, 204, 594

Wheeler G 351

Wheeler GD 264

Whiteneck GG 470

Williams B (deceased) 179 (O)

Woolfenden A 137

Wutthiphan B 696

Yakovleff A 91

Yakura JS 188, 707

Yamada K 493

Yamamoto M 493

Yamanishi T 481

Yang L 611

Yasuda K 481

Ye JH 320

Yone K 403

York DH 39

Yun IG 176

Zach GA 498 (L)

Zanolla L 305

Zarampoukas T 268

Zilvold G 507 